\title{
Matrine Induces Cell Anergy in Human Jurkat T Cells through Modulation of Mitogen-Activated Protein Kinases and Nuclear Factor of Activated T-Cells Signaling with Concomitant Up-Regulation of Anergy-Associated Genes Expression
}

\author{
Ting Li, Vincent Kam Wai Wong, Xiao Qin Yi, Yuen Fan Wong, Hua Zhou, ${ }^{*}$ and Liang Liu* \\ Center for Cancer and Inflammation Research, School of Chinese Medicine, Hong Kong Baptist University; 7 Baptist \\ University Road, Kowloon Tong, Kowloon, Hong Kong, China. \\ Received May 14, 2009; accepted October 5, 2009; published online October 9, 2009
}

\begin{abstract}
Induction of immunotolerance has become a new strategy for treating autoimmune conditions in recent decades. However, so far there is no ideal therapeutics available for clinical use. Medicinal herbs are a promising potential source of immunotolerance inducers. In the current study, we sought first to optimize conditions for a validated cellular model of human Jurkat cells; and then used this model to screen bioactive compounds derived from medicinal plants for inducing $T$ cell anergy in comparison with the effect of well-known $T$ cell anergy inducer, ionomycin. The results showed that passage of the cells, and concentration and stimulation time of ionomycin on the cells could influence the ability of $T$ cell anergy induction. Matrine, a small molecule derived from the root of Sophora flavescens AIT., was demonstrated to be effective in inducing $T$ cell anergy in human Jurkat cells. The cells exposed to matrine showed markedly decreased mRNA expression of interleukin-2, an indicator of $T$ cell anergy, when the cells were stimulated by antigens, anti-OKT3 plus anti-CD28. Mechanistic study showed that ionomycin and matrine could up-regulate the anergy-associated gene expressions of CD98 and Jumonji and activate nuclear factor of activated T-cells (NFAT) nuclear translocation in absence of cooperation of AP-1 in Jurkat cells. Pre-incubation with matrine or ionomycin could also shorten extracellular signal-regulated kinase (ERK) and suppress c-Jun $\mathrm{NH}_{2}$-terminal kinase (JNK) expression on the anergic Jurkat cells when the cells were stimulated with anti-OKT-3 plus anti-CD28 antibodies. Thus, matrine is a strong candidate for further investigation as a $\mathbf{T}$ cell immunotolerance inducer.
\end{abstract}

Key words Jurkat T cell; cell anergy; matrine; herbal medicine; immunotolerance inducer

$\mathrm{T}$ cell activation plays a pivotal role in the pathogenesis of human autoimmune and chronic inflammatory illnesses. In the past decades, great efforts have been made to find immunosuppressants that can intervene in $\mathrm{T}$ cell activation and proliferation. However, in treatment of some refractory cases of these illnesses, immunosuppressants must be taken for long-term, even life-long, in spite of various side effects. Therefore, $\mathrm{T}$ cell immunotolerance induction leading to $\mathrm{T}$ cells anergy has been becoming a promising new therapeutic strategy for autoimmune conditions. Such agents typically have few side effects because they act only as adjuvants in the human body to induce $\mathrm{T}$ cell anergy in response to unknown antigens. In fact, $\mathrm{T}$ cell activation in the body requires engagement of both the T cell receptor (TCR) and CD28 on the cells through participation of the major histocompatibility complex (MHC) peptides and B7 family members on antigen presenting cells (APC). When T cells are stimulated by antigen(s) through TCR in the absence of CD28/B7, the cells develop long-term hyporesponsiveness to subsequent stimulations ${ }^{1}$; this is known as T cell anergy. In other words, $\mathrm{T}$ cell anergy is an immunotolerance condition in which $\mathrm{T}$ cells are intrinsically and functionally inactivated following encounter with antigen(s); the cells remain alive but hyporesponsive for an extended period of time. ${ }^{2)}$ Furthermore, this hyporesponsiveness is characterized by a suppression of interleukin (IL)-2 secretion, such that $\mathrm{T}$ cell activation and proliferation are in turn inhibited. ${ }^{1)}$ Thus, agents that stimulate TCR alone and suppress IL-2 secretion before T cells are activated by antigen(s) are likely ideal candidates for use as $\mathrm{T}$ cell immunotolerance inducers.
Although detailed molecular mechanisms of T cell anergy remain unclear, it is well-known that it is partially mediated through calcineurin and the nuclear factor of activated T-cells (NFAT). ${ }^{3)}$ In the resting T cells, NFAT proteins are phosphorylated and reside in the cytoplasm. When the cells were stimulated, NFAT proteins are dephosphorylated by calcineurin and then translocated into the nucleus and become the transcriptional active, and thus providing a direct line between intracellular $\mathrm{Ca}^{2+}$ signaling and gene expression. ${ }^{4)}$ Especially, when $\mathrm{T}$ cells received TCR/CD28 stimulation, NFAT will be dephosphorylated, translocated into nucleus and cooperated with AP-1, the transcriptional partner of NFAT which is composed of heterodimers of Fos- and Junfamily protein, to induce many genes encoding cytokines, chemokines and other products in the immune responses. Different from NFAT, the activators of AP-1 are mitogen-activated protein kinases (MAPKs) including c-Jun $\mathrm{NH}_{2}$-terminal kinase (JNK), extracellular signal-regulated kinase (ERK) and p38 kinase. In contrast, when NFAT was preactivated by $\mathrm{Ca}^{2+}$ signals or TCR stimulation alone without co-stimulation provided by $\mathrm{CD} 28$ and thus in the absence of its transcriptional factor AP-1, the T cell anergy will be induced. ${ }^{5)}$

To date, the optimum and simple cellular models for investigating the mechanism of $\mathrm{T}$ cell anergy and screening $\mathrm{T}$ cell anergy inducers are still insufficient, although several mouse cellular models have been well-developed. ${ }^{6-8)}$ Using Jurkat $\mathrm{T}$ cells, a human leukemia cell line, a $\mathrm{T}$ cell anergy model was successfully explored, with pre-treatment of the calcium ionophore, ionomycin. The results showed that the Jurkat $\mathrm{T}$ 
cells can be used for the study of T cell anergy induction as well as for evaluation of the potential values of bioactive chemicals in immunotolerance induction. ${ }^{9)}$ In the current study, we used the cellular model of Jurkat cells with slight modifications to screen the candidate $\mathrm{T}$ cell anergy inducers derived from medicinal herbs. As a result, matrine derived from the root of Sophora flavescens AIT. was showed to be active in inducing $\mathrm{T}$ cell anergy through modulation of MAPKs and NFAT signaling with concomitant up-regulation of anergy-associated genes expression.

\section{MATERIALS AND METHODS}

Antibodies and Reagents Ionomycin was purchased from Merck \& Co (Rahway, NJ, U.S.A.). Matrine (Fig. 1A) derived from the root of Sophora flavescens AIT., and artemisinin (Fig. 1B) derived from Artemisia annua L. were obtained from Sigma Chemical Co. (St. Louis, MO, U.S.A.). All were dissolved in dimethyl sulfoxide (DMSO) for cell culture in current experiments. Anti-OKT3 and anti-CD28 antibodies used for activating Jurkat $\mathrm{T}$ cells were purchased from e-Bioscience (San Diego, U.S.A.) and BD Pharmingen (San Diego, U.S.A.), respectively. PCR kits and TRIzol ${ }^{\mathrm{TM}}$ were purchased from Gibco BRL (Gaithersburg, MD, U.S.A.). Primers were synthesized by Sigma Chemical Co. (St. Louis, MO, U.S.A.) and stored at $-80^{\circ} \mathrm{C}$ until use. All working solutions were stored at $-20^{\circ} \mathrm{C}$ for experiments.

Cell Culture The Jurkat $\mathrm{T}$ cell line was obtained from the American Type Culture Collection (ATCC, Rockvill, MD, U.S.A.). In experiments, the cells were cultured in RPMI 1640 supplemented with 10\% fetal bovine serum (FBS), $2 \mathrm{~mm}$ glutamine, $100 \mathrm{U} / \mathrm{ml}$ streptomycin and $100 \mathrm{U} / \mathrm{ml}$ penicillin (complete media). The cells were sub-cultured every $3 \mathrm{~d}$, and the density was maintained at $10^{5} / \mathrm{ml}$.

Induction of Cell Anergy and Stimulation $T$ cell anergy induction was carried out as previously described with modifications. ${ }^{9)}$ In brief, Jurkat T cells were seeded into a 6well plate at $1.25 \times 10^{6} \mathrm{cells} / \mathrm{ml}$, and the cells were then incubated with $1 \mu \mathrm{M}$ ionomycin for $16 \mathrm{~h}$ except specifically indicated times at $37^{\circ} \mathrm{C}$ in an incubator with $5 \% \mathrm{CO}_{2}$. For examination the effect of ionomycin or matrine on the anergyassociated genes of CD98 and Jumonji, the cells were incubated with ionomycin or matrine for $16 \mathrm{~h}$ and then harvested. For detection of IL-2 expression, the pretreatment cells were washed with phosphate buffered saline (PBS) and then stimulated with $10 \mu \mathrm{g} / \mathrm{ml}$ of anti-OKT3 and anti-CD28 antibodies for another $4 \mathrm{~h}$.

RNA Extraction Total RNA was extracted from the cultured cells using TRIzol according to the manufacturer's instructions. In brief, the cells were re-suspended in $1.0 \mathrm{ml}$
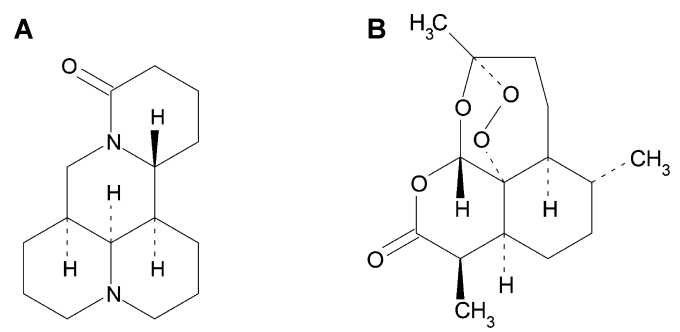

Fig. 1. Chemical Structures of Matrine (A) and Artemisinin (B)
TRIzol reagent and incubated for $5 \mathrm{~min}$ at room temperature. After incubation, $0.2 \mathrm{ml}$ of chloroform was added to the TRIzol. The mixtures were vortexed and then incubated for $3 \mathrm{~min}$ at room temperature. Following this incubation, the mixtures were centrifuged at $12000 \mathrm{~g}$ for $15 \mathrm{~min}$, and then the aqueous layer was transferred into another tube. After centrifugation at $12000 \mathrm{~g}$ for $10 \mathrm{~min}$, the RNA was precipitated from the aqueous phase by isopropyl. The RNA pellet was washed twice with $75 \%$ ethanol and then dissolved in $0.1 \%$ sterile diethylpyrocarbonate (DEPC)-water.

Reverse Transcription-Polymerase Chain Reaction (RT-PCR) Analysis Reverse transcription was performed by using the M-MLV First-Strand Kit according to the manufacturer's instructions, together with $5 \mu \mathrm{g}$ of the total RNA. And then, PCR analysis was performed by using $2 \mu \mathrm{l}$ cDNA, DEPC-water, $1 \times$ PCR buffer, $0.5 \mathrm{~mm}$ deoxynucleotide triphosphate (dNTP) mixture, $10 \mathrm{pmol}$ of each primer and 0.5 units of Taq polymerase. The total volume of PCR reaction was $25 \mu \mathrm{l}$. The primers were showed as follows:

IL-2: sense 5'-ACTCACCAGGATGCTCACAT-3'; antisense 5'-AGGT-AATCCATCTGTTCAGA-3';

Jumonji: sense 5'-GCTCAGGACTTACGGAAACA-3'; antisense 5'-TGTGGTTGACAGCGGAACTG-3';

CD98: sense 5'-GCTGCTGCTCTTCTGGCTC-3'; antisense 5'-GCCAGTGGCATTCAAATAC-3';

Glyceraldehyde-3-phosphate dehydrogenase (GAPDH): sense 5'-GGTCTTACTCCTTGGAGGCCATGT-3'; antisense 5'-ACCTAACTACATGGTTTACATGTT-3'.

Western Blotting Analysis For NFAT nuclear translocation assay, the human Jurkat cells were incubated with ionomycin $(1 \mu \mathrm{M})$ or matrine $(500 \mu \mathrm{M})$ for different indicated time points and then the cells were harvested and washed by PBS twice. The nuclear proteins of cells were then prepared using NucBuster ${ }^{\mathrm{TM}}$ Reagents (Novagen, U.S.A.) according to manufacturer's instructions. In brief, the washed cells were resuspended using $60 \mu \mathrm{l}$ NucBuster ${ }^{\mathrm{TM}}$ Reagents 1 per $30 \mu \mathrm{l}$ of the packed cells and processed twice by vortexing for $15 \mathrm{~s}$, incubated on ice for $5 \mathrm{~min}$, vortexed again for $15 \mathrm{~s}$, and finally centrifuged at $16000 \mathrm{~g}$ for $5 \mathrm{~min}$. The supernatants, which contained cytoplasmic proteins, were discarded; the cell pellets were resuspended in $30 \mu \mathrm{l}$ of NucBuster ${ }^{\mathrm{TM}}$ Reagents Extraction Regent 2. The same vortexing, icing and repeated vortexing procedures were repeated once to prepare the nuclear proteins of the cells. Equal amounts of the nuclear proteins were analyzed by $8 \%$ sodium dodecyl sulfate-polyacrylamide gel electrophoresis (SDS-PAGE), and then the proteins were electro-transferred onto nitrocellulose membrane. The nitrocellulose membrane was blocked by $5 \%$ dried milk for $60 \mathrm{~min}$ and then incubated with NFAT (BD Bioscience) and lamin B (Santa Cruz) overnight at $4{ }^{\circ} \mathrm{C}$. Next day, the membrane was washed with Tris-buffered saline containing Tween20 (TBS-T) again and further incubated with horseradish peroxidase (HRP)-conjugated secondary antibodies for $60 \mathrm{~min}$. The blots were developed by using ECL Western Blotting Dection reagents (Amersham Bioscience).

For examinating the effect of drugs on MAPK signaling of the cells, the phosphorylation forms of JNK (P-JNK), total JNK; phosphorylation forms of ERK 1/2 (P-ERK1/2), total ERK; phosphorylation forms of p38 (P-p38) and total p38 kinases from whole cellular proteins were detected. Briefly, the cells were pre-incubated with ionomycin $(1 \mu \mathrm{M})$ or ma- 
trine $(500 \mu \mathrm{M})$ for $16 \mathrm{~h}$ and then co-stimulated with antiOKT3 plus anti-CD28 (10 $\mu \mathrm{g} / \mathrm{ml}$ each) for different indicated time points and finally harvested or directly harvested without stimulation. The harvested cells were lysed with lysis buffer containing $50 \mathrm{~mm}$ Tris- $\mathrm{HCl}$, pH 7.5, $250 \mathrm{~mm} \mathrm{NaCl}$, $5 \mathrm{~mm}$ EDTA, $1 \mathrm{~mm}$ DTT, $1 \%$ Triton, $50 \mathrm{~mm} \mathrm{NaF,} 1 \mathrm{~mm}$ sodium orthovanadate, $0.5 \mathrm{~mm}$ PMSF and $1 \times$ protease inhibitor mix (Roche, U.S.A.) to produce whole celluar proteins. Equal amounts of whole-cell lysates were analyzed by $10 \%$ SDS-PAGE and transferred to the nitrocellulose membrane as mentioned above. The primary antibodies used in this study were rabbit antibodies specific for P-p38, P-JNK (Thr183/Try185)，JNK，P-ERK1/2 (Thr220/Try204) and ERK (Cell Signaling), and mouse antibodies specific for $\beta$ actin (BD Bioscience) and p38 (Santa Cruz).

Cytotoxicity Assay The cytotoxicity of matrine with different concentrations was determined by using 3-(4,5-dimethylthiazol-2-yl)-2,5-diphenyl tetrazolium bromide (MTT) assay as described previously. ${ }^{10)}$ Briefly, Jurkat $\mathrm{T}$ cells $\left(10^{5}\right.$ cells/well $)$ were cultured with or without matrine in triplicate in a 96-well flat-bottomed plate (Costar, Corning Incorporated, Corning, NY, U.S.A.) for $72 \mathrm{~h}$. MTT $(5 \mathrm{mg} / \mathrm{ml})$ was added for $4 \mathrm{~h}$ incubation, and then solvent (10\% SDS, $50 \% \mathrm{~N}, \mathrm{~N}$-dimethy formamide, $\mathrm{pH} 7.2$ ) was added to dissolve the purple precipitate. $A_{570 \mathrm{~nm}}$ was determined from each well on the next day. Percentage of cell viability was calculated using the following formula: cell viability $(\%)=A_{\text {treated }}$ $A_{\text {control }} \times 100$. Data were obtained from three independent experiments.

\section{RESULTS}

Validation of $\mathbf{T}$ Cell Anergy Induction Model in Human Jurkat Cells To validate the method for inducing human $\mathrm{T}$ cell anergy in Jurkat cells, we firstly examined the optimum concentration of ionomycin for inducing the cell anergy. As the hallmark of T cell clonal anergy is a decrease of IL-2 expression, ${ }^{11)}$ mRNA expression of IL-2 in the cells pre-treated with or without ionomycin for $16 \mathrm{~h}$ was determined by using semi-quantitative RT-PCR analysis after $4 \mathrm{~h}$ co-stimulation of $10 \mu \mathrm{g} / \mathrm{ml}$ of anti-OKT3 and anti-CD28 antibodies. The results showed that pre-treatment with ionomycin significantly reduced the mRNA expression of IL-2 cytokine of the Jurkat $\mathrm{T}$ cells induced by co-stimulation of anti-OKT3 plus anti-CD28 antibodies, especially at a $1.0 \mu \mathrm{M}$ concentration of ionomycin (Fig. 2A). This is consistent with the previous reports. ${ }^{9)}$ Accordingly, we used ionomycin at $1.0 \mu \mathrm{M}$ concentration for inducing $\mathrm{T}$ cell anergy in following test.

To determine the time kinetics of ionomycin stimulation, we pretreated the cells with ionomycin for different time periods. The results showed that IL-2 mRNA expression of the cells pretreated by ionomycin started to decrease at $4 \mathrm{~h}$ and reached the maximum decrease from 8 to $20 \mathrm{~h}$ under co-stimulation of anti-OKT3 and anti-CD28 antibodies (Fig. 2B). And then the potency of ionomycin for induction of $\mathrm{T}$ cell anergy diminished after $20 \mathrm{~h}$. A previous report showed that pre-treatment of $16 \mathrm{~h}$ was effective for inducing cell anergy, ${ }^{3,12)}$ which is in line with our present results. Thus, $16 \mathrm{~h}$ of pre-treatment of ionomycin was used in following studies.

To determine whether different passages of the cells have

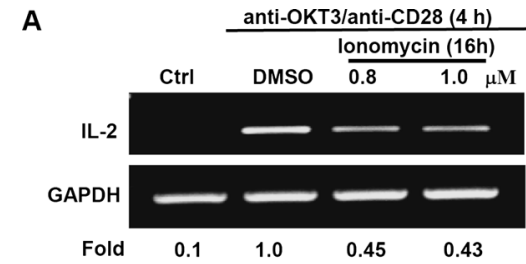

B

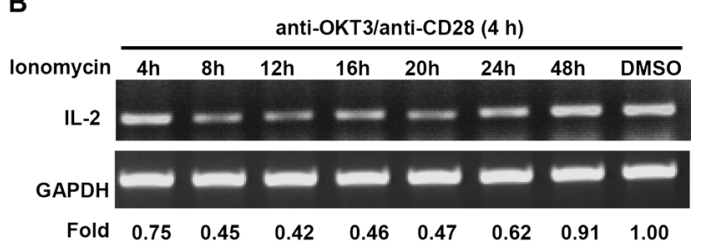

C

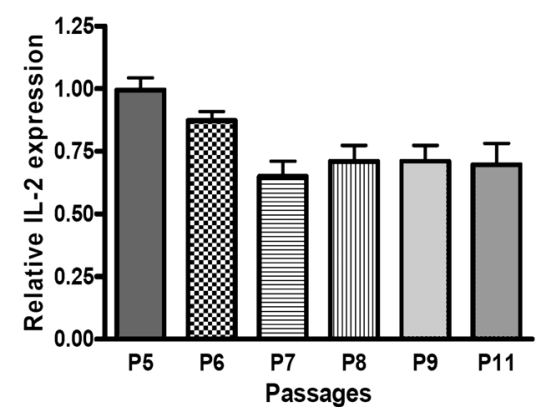

Fig. 2. (A) mRNA Expression of IL-2 in the Anergized Jurkat T Cells Induced by Pre-treatment of Ionomycin

Human Jurkat T cells were either treated with DMSO as control, or incubated with $0.8 \mu \mathrm{M}$ or $1.0 \mu \mathrm{M}$ ionomycin for $16 \mathrm{~h}$, and then costimulated with anti-OKT3 and antiCD28 for $4 \mathrm{~h}$.

(B) Anergic Effect of Ionomycin on IL-2 mRNA Expression in the Jurkat T Cells

Human Jurkat T cells were pre-treated with $1.0 \mu \mathrm{m}$ ionomycin for the indicated time durations and treated with anti-OKT3 and anti-CD28 for $4 \mathrm{~h}$.

(C) Suppressive Potency of Ionomycin on the mRNA Expression of IL-2 in Different Passages of the Human Jurkat T Cells

The folds of expression over DMSO were indicated.

distinct potencies in responding to $\mathrm{T}$ cell anergy induction, we examined the levels of IL-2 mRNA expression in different passages of the Jurkat cells by pre-treatment of ionomycin for $16 \mathrm{~h}$ and then stimulation with anti-OKT3 plus anti-CD28 for $4 \mathrm{~h}$. In our study, Jurkat $\mathrm{T}$ cells purchased from ATCC were marked as passage 1 and then cultured under our cell culture system at an interval of $3 \mathrm{~d}$ for one passage. Passage 5 to passage 11 of the cells were examined for evaluating their capabilities of inducing the cell anergy by pre-treatment of $1.0 \mu \mathrm{M}$ ionomycin for $16 \mathrm{~h}$, followed by the co-stimulation of anti-OKT3 and anti-CD28 antibodies for $4 \mathrm{~h}$. The results shown in Fig. 2C demonstrated that mRNA expression of IL-2 in all passages of the cells was reduced to certain extent, with passages 7 to 11 of cells showing an obvious reduction. This result suggested that human Jurkat cells from passage 7 to 11 are more appropriate for the study of $\mathrm{T}$ cell anergy induction.

The Potency of Matrine on Inducing IL-2 Reduction in Human Jurkat Cells $\mathrm{T}$ cell anergy induced by ionomycin is related to sustain low-level signaling through the $\mathrm{Ca}^{2+}$ / calineurin/NFAT pathway. ${ }^{12)}$ Several compounds derived from medicinal herbs, such as matrine and artemisinin, have been reported effective in activating calcium flux in myocytes. ${ }^{13)}$ 
Thus, we predominantly tried to determine whether these compounds pretreatment could reduce IL-2 mRNA expression on human Jurkat $\mathrm{T}$ cells under co-stimulation of antiOKT3 and anti-CD28 antibodies.

Matrine, a compound derived from the dried roots of Sophora flavescens Aiт., has been used for a long time in China to treat viral hepatitis, cancer, cardiac problems and skin diseases. ${ }^{14)}$ We examined the potency of matrine on $\mathrm{T}$ cell anergy induction by determining the level of IL-2 mRNA expression in Jurkat cells. The cells were incubated with matrine at different concentrations and then stimulated by antiOKT3 and anti-CD28 antibodies. The results showed that matrine could significantly suppress IL-2 mRNA expression of the cells in a dose-dependent manner (Fig. 3A). Cytotoxicity assay showed that matrine had no significant cytotoxicity within the range of effective drug concentrations used (Fig. $3 \mathrm{C})$. These results indicated that the suppressive effect of matrine on IL-2 mRNA expression in Jurkat T cells did not result from its cytotoxicity. Another compound, artemisinin derived from the medicinal herb Artemisia annua L. demonstrated its potency in anti-inflammation, immune regulation and enhancement of $\mathrm{Ca}^{2+}$ flux, ${ }^{13,15)}$ and it has been used in many countries for treatment of severe and chloroquineresistant malaria. However, this compound showed no ability to induce $\mathrm{T}$ cell anergy (Fig. 3B).

The Effect of Ionomycin and Matrine on NFAT Nu- clear Translocation in Human Jurkat Cells T cell anergy depends on sustained low-level signaling through $\mathrm{Ca}^{2+} /$ calineurin/NFAT pathway. NFAT activation and nuclear translocation could be achieved with sustained levels of $\mathrm{Ca}^{2+}$ mobilization induced by $\mathrm{Ca}^{2+}$ ionophore ionomycin. ${ }^{12,16)}$ In our current study, we investigated whether or not ionomycin alone could induce NFAT nuclear translocation in Jurkat cells. The results demonstrated that ionomycin alone could obviously increase NFAT nuclear translocation (Fig. 4A, left panel). In light of the inhibitory effect of matrine on IL-2 mRNA expression, we further determined whether matrine possesses a similar property as ionomycin did, i.e., to activate NFAT nuclear translocation and then reduce IL-2 mRNA expression, the hallmark of $\mathrm{T}$ cell anergy. The results shown in Fig. 4A (right panel) indicate that matrine could induce NFAT nuclear translocation as well.

The Effects of Ionomycin and Matrine on MAPKs Activation without Stimulation of Anti-OKT-3 Plus AntiCD28 in Human Jurkat Cells It was reported that NFAT was prevented from interacting with its transcription partner AP-1 (Fos/Jun) in the anergic T cells. ${ }^{17)}$ We therefore investigated the effect of ionomycin alone on the activation of MAPKs which activate AP-1. The results showed that ionomycin alone, i.e., without stimulation of anti-OKT-3 plus anti-CD28, has no significant effect to activate MAPKs (Fig. 4B, left panel). This indicates that ionomycin may induce
A

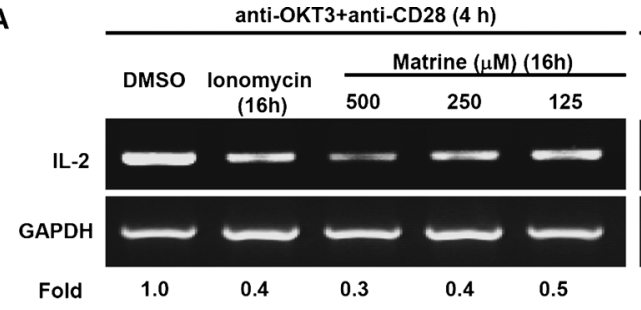

B

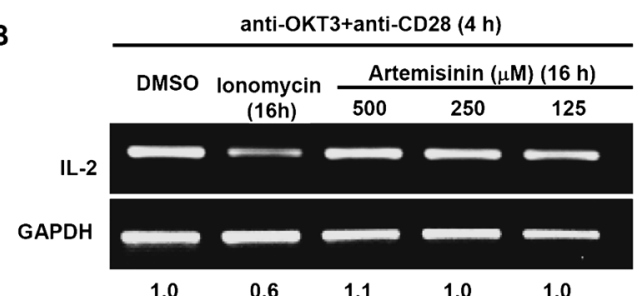

C

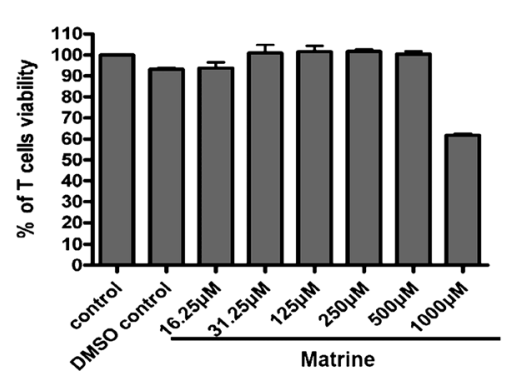

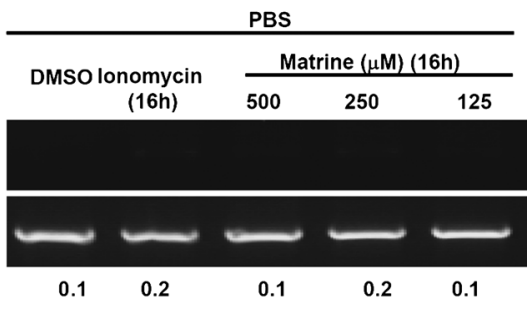

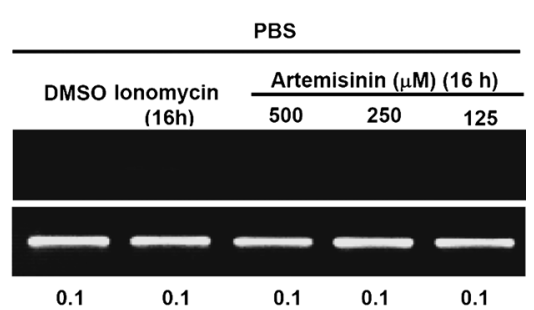

Fig. 3. (A, B) Potency of Matrine and Artemisinin on Induction of T Cell Anergy in Human Jurkat Cells

Human Jurkat T cells were pre-treated with DMSO (control), $1.0 \mu \mathrm{M}$ ionomycin and the indicated concentrations of matrine (A) or artemisinin (B) for $16 \mathrm{~h}$. On the next day, the cells were washed with PBS and then co-stimulated with anti-OKT3 plus anti-CD28 antibodies for $4 \mathrm{~h}$. Total RNA was isolated and the RT-PCR was performed using primers specific for IL-2 and GAPDH.

(C) Cytotoxicity Assay of Matrine on Human Jurkat T Cells

The cells were treated by matrine at the indicated concentrations for $3 \mathrm{~d}$, and MTT reagent was then added to the cells for $4 \mathrm{~h}$ of further incubation followed by the addition of solubilization buffer. 
A

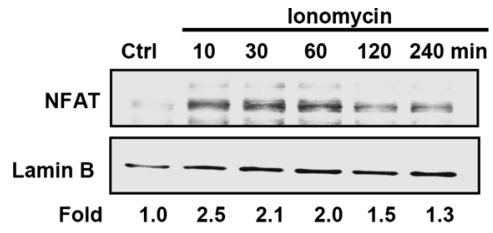

B

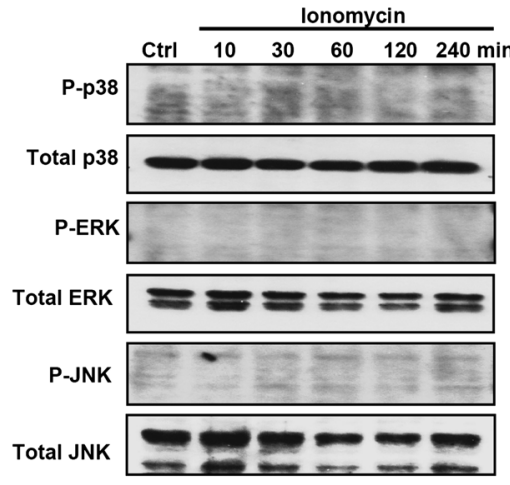

C
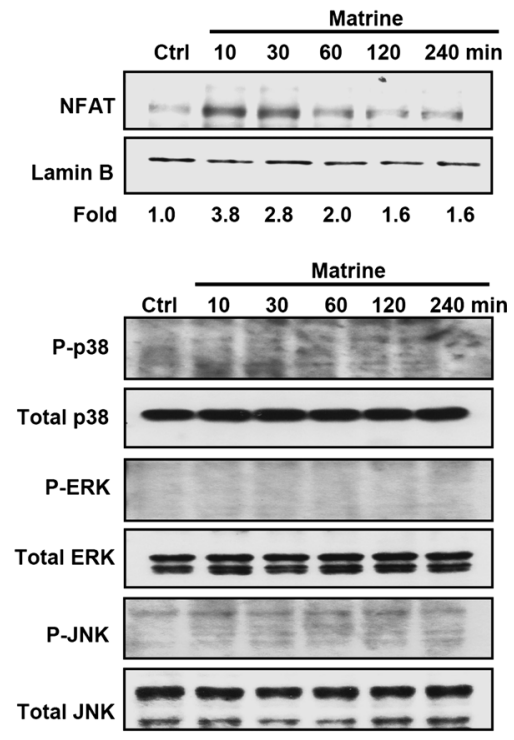

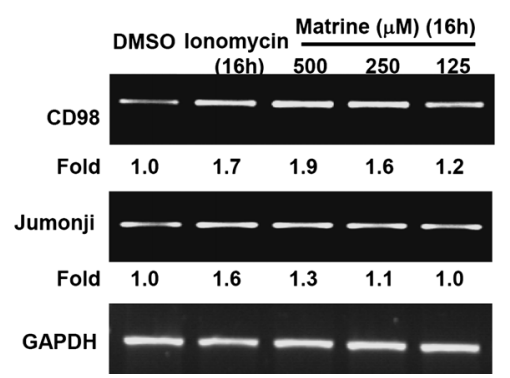

Fig. 4. (A) Time Kinetics of Ionomycin and Matrine on NFAT Nuclear Translocation in the Cells

The cells were incubated with $1 \mu \mathrm{M}$ ionomycin (A, left panel) or $500 \mu \mathrm{M}$ matrine (A, right panel) for the indicated time intervals. And then, nuclear proteins were prepared and analyzed by Western blotting using antibody against NFAT. The control cells were treated with equal volume of medium.

(B) Time Kinetics of Ionomycin and Matrine on Influences of MAPKs Phosphorylation in Jurkat T Cells without Stimulation of Anti-OKT-3 and Anti-CD28

The cells were incubated with $1 \mu \mathrm{M}$ ionomycin (B, left panel) or $500 \mu \mathrm{M}$ matrine (B, right panel) for the indicated time intervals. And then, the cells were lysed and subjected to Western blotting analysis using antibodies against the total forms of ERK, JNK and p38 and phosphorylation forms of ERK, JNK and p38. The control cells were treated with equal volume of medium.

(C) Up-Regulatory Effects of Matrine and Ionomycin on Anergy-Associated Genes CD98 and Jumonji Expression in the Anergic Human Jurkat T Cells

Human Jurkat T cells were pre-treated with DMSO (control), ionomycin or with matrine at the indicated drug concentrations for $16 \mathrm{~h}$. On the next day, total RNA of the cells was isolated and the RT-PCR was performed using primers specific for CD98, Jumonji and GAPDH. The folds of expression over DMSO were indicated.

human Jurkat cell anergy through activation of NFAT, but without affecting on the MAPKs signaling of the cells. We proposed that matrine alone might have the same effect on MAPKs activation and then investigated the effect with Western blotting. As we expected, matrine alone showed no significant differences on the activation of MAPKs compared to the control group (Fig. 4B, right panel).

Ionomycin and Matrine Up-Regulate CD98 and Jumonji mRNA Expression Previous report showed that a set of anergy-associated genes, such as the cellular surface gene CD98 and the transcriptional gene Jumonji, are up-regulated during ionomycin-induced T cell anergy. ${ }^{12)}$ We therefore examined the effect of matrine or ionomycin alone on the expression of anergy-associated genes, CD98 and Jumonji. The results showed expression of genes CD98 and Jumonji to be up-regulated in the ionomycin and matrinetreated Jurkat cells. And, this effect was in a dose-dependent manner at the concentrations of matrine from 125 to $500 \mu \mathrm{M}$ (Fig. 4C).

The Effects of Ionomycin and Matrine on MAPKs Expression on Human Jurkat Cells Activated by Anti-OKT3 Plus Anti-CD28 The ERK pathway of the cells could be preferentially impaired in rapamycin-induced $\mathrm{T}$ cell anergy. ${ }^{18)}$ So, we further examined whether MAPKs pathway activation is suppressed during the anergic status of Jurkat $\mathrm{T}$ cells. The ERK, JNK and p38 was determined by Western blot in the anergic Jurkat T cells. The results showed that costimulation of anti-OKT3 plus anti-CD28 antibodies resulted in significant ERK, JNK and p38 phosphorylation in Jurkat cells; while the ionomycin and matrine-mediated anergic cells showed no significant change in the phosphorylation of p38. However, ionomycin and matrine demonstrated a similar influence on ERK and JNK phosphorylation, leading to a marked shortage of ERK activation and suppressed JNK expression (Figs. 5A \& B).

\section{DISCUSSION}

It has been well-documented that stimulation of $\mathrm{T}$ cells through TCR without co-stimulatory molecule can result in hyporesponsiveness to the subsequent antigens, named as $\mathrm{T}$ cell anergy induction. However, ideal in vivo and in vitro models for the study of $\mathrm{T}$ cell anergy are still lacking. Human Jurkat cells have been reported to undergo $\mathrm{T}$ cell an- 


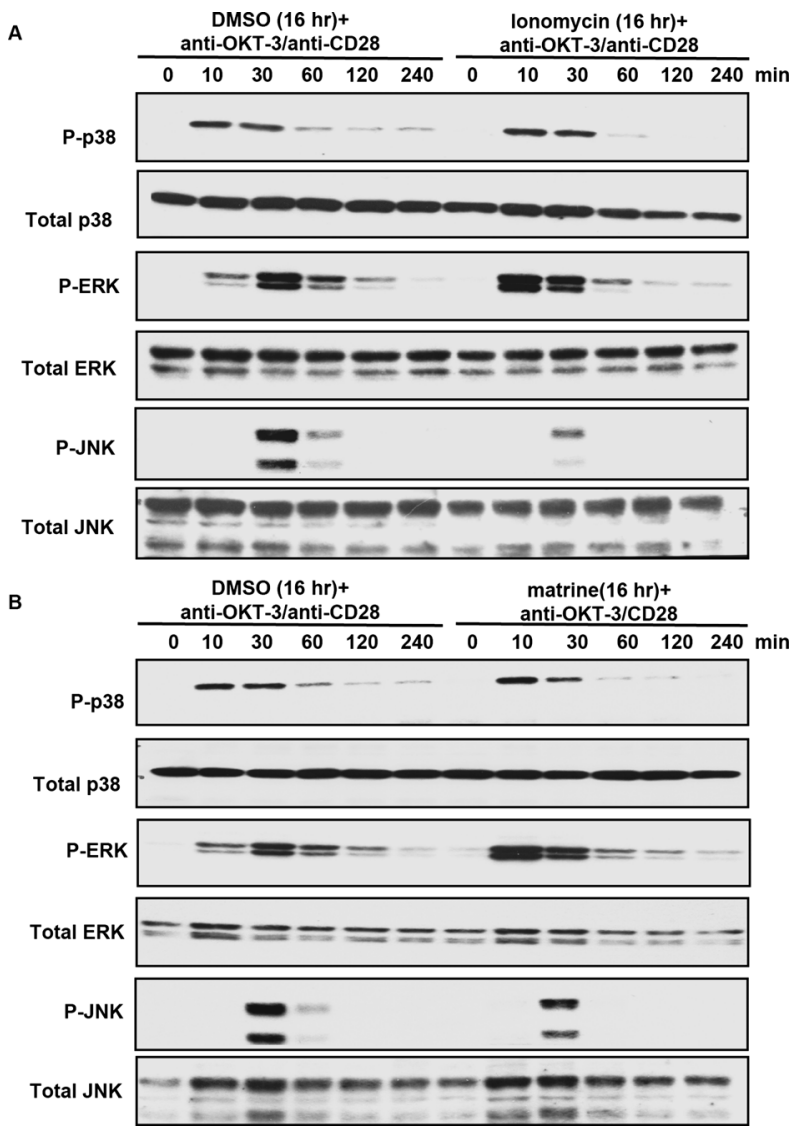

Fig. 5. Effects of Ionomycin and Matrine on the Phosphorylation of MAPKs Activated by Anti-OKT3 Plus Anti-CD28 Antibodies on the Anergic Human Jurkat T Cells

Cells were incubated with $1.0 \mu \mathrm{M}$ ionomycin (A) or $500 \mu \mathrm{M}$ matrine (B) for $16 \mathrm{~h}$, and then they were washed with PBS after incubation and then followed by stimulation with anti-OKT3 plus anti-CD28 antibodies for different indicated time points. Finally, the cells were lysed and subjected to Western blotting analysis using antibodies against the total form of ERK, JNK and p38 and phosphorylation form of ERK, JNK and p38.

ergy using ionomycin, ${ }^{9}$ ) a common anergy inducer, ${ }^{3,12,19,20)}$ while the methods need to be further validated with precise modeling conditions according to different conditions of different laboratories, such as the proper responding passages of the cells, and the optimum concentration and treatment time of ionomycin on the cells. ${ }^{9)}$ In the current study, we first tried to validate the method by examining effects of a range of ionomycin concentrations, stimulation periods, and cell passages because cell lines from different laboratories may have distinct characteristic. And then, we used the validated methods to screen the effective compounds derived from the medicinal plants. Consequently, the results showed that $\mathrm{T}$ cell anergy induction in human Jurkat cells depends in some degrees on the passage of cell. For instance, passages $7-11$ of the cells might be suitable for cell anergy induction purpose because these 5 passages of the cells showed markedly decrease in producing IL-2 mRNA evoked by the co-stimulation of anti-OKT3 plus anti-CD28 antibodies. Furthermore, cells treated with $1.0 \mu \mathrm{M}$ ionomycin for 8 to $20 \mathrm{~h}$ was shown to be effective in inducing $\mathrm{T}$ cell anergy. However, taken the experiences of similar works done in other laboratories together, ${ }^{3,12)}$ we decided to employ $16 \mathrm{~h}$ of ionomycin and matrine incubation in the cell culture for evaluating the drug actions.
Using this validated methods, we examined the potency of matrine and artemisinin derived from two medicinal herbs in inducing $\mathrm{T}$ cell anergy. We found that matrine, but not artemisinin, could effectively induce $\mathrm{T}$ cell anergy just as ionomycin does, showing marked decrease of IL-2 mRNA expression after co-stimulated by anti-OKT3 plus anti-CD28 antibodies. This result suggests that matrine might have potential for further development as a novel T cell anergy inducer. Surprisingly, artemisinin and its derivatives have been proven potent for anti-inflammation and immunoregulation, ${ }^{21)}$ and they have been already used to treat autoimmune conditions, like systemic lupus erythematosus (SLE) and allergic contact dermatitis. ${ }^{22)}$ However, artemisinin showed no potency in $\mathrm{T}$ cell anergy induction in our study. Thus, artemisinin may function as an immunosuppressant but not as a $\mathrm{T}$ cell anergy inducer.

In fact, $\mathrm{Ca}^{2+} /$ calcineurin-NFAT-mediated signaling pathway is involved in diverse cellular reactions by regulating gene expression either positively or negatively. Interaction of NFAT with AP-1 turns on the genes involved in active immune responses, while NFAT without cooperative binding of AP-1 turns on a $\mathrm{T}$ cell anergy program. ${ }^{23)}$ In other words, sustained calcium signaling induces $\mathrm{T}$ cell anergy which is mediated through calcineurin and the transcription factor of NFAT. In general, NFAT occurs in response to $\mathrm{Ca}^{2+}$ signals or TCR stimulation alone. Therefore, in our current study, the $\mathrm{Ca}^{2+}$ ionophore, ionomycin, was used to induce anergy on human Jurkat cells at $1.0 \mu \mathrm{M}$ concentration, and ionomycin alone could also activate NFAT nuclear translocation of the cells. It is mentioned that NFAT activation could induce the cell anergy in the absence of its transcription factor partner AP-1. ${ }^{23)}$ MAPKs activation contributes to the synthesis and activation of Fos and Jun, the components of the heterodimeric transcription factor AP-1. ${ }^{24)}$ In our present study, we found that ionomycin alone had no significant effect to activate MAPKs, which means that ionomycin induced NFAT nuclear translocation without cooperating with AP-1. Previous study showed that matrine could activate $\mathrm{Ca}^{2+}$ flux as ionomycin did, interestingly we found that matrine has similar effect of ionomycin to activate NFAT nuclear localization without affecting MAPKs signaling.

Anergized T cells express a novel set of anergy-associated genes, and those genes are grouped into five categories: transcription factors, proteolysis factors, receptors, non-receptor tyrosine phosphatases, and cell surface receptor genes based on specific functions. ${ }^{12)}$ CD98, cell surface receptor genes, and Jumonji, transcriptional gene, may function in suppression of IL-2 mRNA expression. And both of two genes were found to be up-regulated in human Jurkat T cells induced by ionomycin or matrine alone with the RT-PCR technique. The anergic state of Jurkat cells induced by ionomycin and matrine shares some similarities with that of other anergized cells. ${ }^{18,25)}$

Furthermore, T cell anergy model induced by rapamycin was reported to mediate through suppression of ERK pathway. ${ }^{26)}$ In fact, the results exhibit that both of ionomycin and matrine significantly could shorten ERK phosphorylation in co-stimulated cells without changing the trend, indicating that the anergic mechanisms of ionomycin or matrineinduced $\mathrm{T}$ cell anergy might be different from the $\mathrm{T}$ cell anergy induced by rapamycin. Furthermore, it has been re- 
ported that JNK activation required a second signal provided by co-stimulatory molecules. ${ }^{27,28)}$ Our results demonstrate that the phosphorylation of JNK was suppressed in the anergic Jurkat $\mathrm{T}$ cells induced by ionomycin or matrine when the cells were co-stimulated by anti-OKT3 plus anti-CD28 antibodies. It is indicated that both of matrine and ionomycin pretreatment might have potential to prevent the co-stimulatory signaling provided by anti-CD28 antibody. Moreover, JNK serves as an NFAT kinase and phosphorylates NFAT. ${ }^{29)}$ As mentioned above, NFAT could be dephosphorylated and translocated into nuclear when the cells enter anergic program. Therefore, we proposed that matrine or ionomycin pretreatment could suppress JNK signaling in the cells and NFAT was inefficiently removed from the nucleus, resulting in nuclear NFAT accumulation, and then further enhanced the anergy development of the cells.

This study presents evidence for five important conclusions with regard to human Jurkat cells: 1) T cell anergy can be effectively induced by ionomycin in human Jurkat T cells by optimizing modeling conditions; the choice of passage of cells, the concentrations and stimulation time of ionomycin in pre-treatment of cells. 2) Matrine, a small molecule derived from a medicinal herb, has been found to be effective in $\mathrm{T}$ cell anergy induction, showing the ability to suppress IL-2 mRNA expression after the cells were co-stimulated by anti-OKT3 plus anti-CD28. 3) The underlying mechanisms of Jurkat $\mathrm{T}$ cell anergy induced by ionomycin or matrine are mediated by NFAT nuclear translocation in the absence of AP-1 cooperation. 4) The genes IL-2, CD98 and Jumonji can be used as indicators of T cell anergy in human Jurkat cells. IL-2 mRNA expression is decreased in the anergized cells, while the genes of CD98 and Jumonji can be up-regulated in the model. 5) Both of ionomycin and matrine have the ability to shorten ERK activation and suppress JNK activation when the cells were co-stimulated by anti-OKT3 plus anti-CD28. More works are still needed to elucidate the functional roles of CD98 and Jumonji in T cell anergy induction and to define the action of matrine in vivo and the precise molecular mechanisms of matrine's action. This work hopefully brings us closer to find better therapeutics for treating autoimmune conditions.

Acknowledgments We wish to thank Dr. Martha Dahlen for her careful checking of the English language on this paper. This work was supported by a Grant from the Research Grants Council of Hong Kong (HKBU 2144/03M).

\section{REFERENCES}

1) Schwartz R. H., J. Exp. Med., 184, 1-8 (1996).

2) Schwartz R. H., Annu. Rev. Immunol., 21, 305-334 (2003).

3) Heissmeyer V., Macian F., Im S. H., Varma R., Feske S., Venuprasad K., Gu H., Liu Y. C., Dustin M. L., Rao A., Nat. Immunol., 5, 255265 (2004).

4) Hogan P. G., Chen L., Nardone J., Rao A., Genes Dev., 17, 2205 2232 (2003).

5) Macian F., Lopez-Rodriguez C., Rao A., Oncogene, 20, 2476-2489 (2001).

6) Jenkins M. K., Chen C. A., Jung G., Mueller D. L., Schwartz R. H., J. Immunol., 144, 16-22 (1990).

7) Williams M. E., Shea C. M., Lichtman A. H., Abbas A. K., J. Immunol., 149, 1921-1926 (1992).

8) Agarwal S., Rao A., Immunity, 9, 765-775 (1998).

9) Howe C. J., LaHair M. M., Robinson P. J., Rodriguez-Mora O., McCubrey J. A., Franklin R. A., Assay Drug Dev. Technol., 1, 537-544 (2003).

10) Gerlier D., Thomasset N., J. Immunol. Methods, 94, 57-63 (1986).

11) Schwartz R. H., Science, 248, 1349-1356 (1990).

12) Macian F., Garcia-Cozar F., Im S. H., Horton H. F., Byrne M. C., Rao A., Cell, 109, 719-731 (2002).

13) Ai J., Gao H. H., He S. Z., Wang L., Luo D. L., Yang B. F., Acta Pharmacol. Sin., 22, 512-515 (2001).

14) Liu J. Y., Hu J. H., Zhu Q. G., Li F. Q., Wang J., Sun H. J., Int. Immunopharmacol., 7, 816-823 (2007).

15) Nagamune K., Beatty W. L., Sibley L. D., Eukaryot. Cell, 6, 2147 2156 (2007).

16) Dolmetsch R. E., Lewis R. S., Goodnow C. C., Healy J. I., Nature (London), 386, 855-858 (1997).

17) Borde M., Barrington R. A., Heissmeyer V., Carroll M. C., Rao A., Immunol. Rev., 210, 105-119 (2006).

18) Chiodetti L., Choi S., Barber D. L., Schwartz R. H., J. Immunol., 176 2279-2291 (2006).

19) Jenkins M. K., Pardoll D. M., Mizuguchi J., Chused T. M., Schwartz R. H., Proc. Natl. Acad. Sci. U.S.A., 84, 5409-5413 (1987).

20) Hayashi R. J., Loh D. Y., Kanagawa O., Wang F., J. Immunol., 160, 33-38 (1998).

21) Noori S., Naderi G. A., Hassan Z. M., Habibi Z., Bathaie S. Z., Hashemi S. M., Int. Immunopharmacol., 4, 1301-1306 (2004).

22) Tam L. S., Gladman D. D., Hallett D. C., Rahman P., Urowitz M. B., J. Rheumatol., 27, 2142-2145 (2000).

23) Im S. H., Rao A., Mol. Cells, 18, 1-9 (2004).

24) Borner C., Warnick B., Smida M., Hartig R., Lindquist J. A., Schraven B., Hollt V., Kraus J., J. Immunol., 183, 882-889 (2009).

25) Fields P. E., Gajewski T. F., Fitch F. W., Science, 271, 1276-1278 (1996).

26) Powell J. D., Lerner C. G., Schwartz R. H., J. Immunol., 162, 27752784 (1999).

27) Su B., Jacinto E., Hibi M., Kallunki T., Karin M., Ben-Neriah Y., Cell, 77, 727-736 (1994).

28) Tamma S. M., Kalyanaraman V. S., Pahwa S., Dominguez P., Modesto R. R., J. Leukoc. Biol., 73, 682-688 (2003).

29) Dong C., Davis R. J., Flavell R. A., Annu. Rev. Immunol., 20, 55-72 (2002). 